\title{
Analysis of an Optically Injected Semiconductor Laser for Microwave Generation
}

\author{
Sze-Chun Chan, Member, IEEE
}

\begin{abstract}
The nonlinear dynamical period-one oscillation of an optically injected semiconductor laser is investigated analytically. The oscillation is commonly observed when the injection is moderately strong and positively detuned from the Hopf bifurcation boundary. The laser emits continuous-wave optical signal with periodic intensity oscillation. Since the oscillation frequency is widely tunable beyond the relaxation oscillation frequency, the system can be regarded as a high-speed photonic microwave source. In this paper, analytical solution of the oscillation is presented for the first time. By applying a two-wavelength approximation to the rate equations, we obtain mathematical expressions that characterize the oscillation. The analysis explains the physical origin of the periodic intensity oscillation as the beating between two wavelengths, namely, the injected wavelength and the cavity resonance wavelength. As the injection strength increases, the optical gain reduces, the cavity is red-shifted through the antiguidance effect, and so the beat frequency increases continuously. The theoretical analysis is useful for designing the system for photonic microwave applications.
\end{abstract}

Index Terms-Injection-locked oscillators, microwave generation, nonlinear dynamics, optical injection, semiconductor lasers.

\section{INTRODUCTION}

$\mathbf{S}$ INGLE-MODE semiconductor lasers subject to constant optical injection have been of great interest in microwave photonics [1]-[9]. Under different operating conditions, the laser exhibits a number of dynamical states such as stable locking, four-wave mixing, period-one oscillation, period-two oscillation, quasi-periodic oscillation, and chaotic oscillation. The simplest state of stable locking has been applied for modulation bandwidth enhancement, chirp reduction, and noise suppression; while the most complicated state of chaotic oscillation has been used in secure communication and chaotic ranging [10]-[12].

In between the two extremes lies the period-one oscillation state. The state is typically found in a large region of the parameter space, where the injection strength is moderately strong and the injection frequency is positively detuned from the so-called Hopf bifurcation boundary. In the period-one state, the output intensity of the laser exhibits high-speed single-period oscillation. The oscillation frequency can be continuously

Manuscript received April 26, 2009; revised June 26, 2009. Current version published February 24, 2010. This work was supported by a Grant from City University of Hong Kong (Project No. 7200110) and a Grant from the Research Grant Council of Hong Kong, China (Project No. CityU 111308).

The author is with the Electronic Engineering Department, City University of Hong Kong, Hong Kong, China (e-mail: scchan@ @ityu.edu.hk).

Color versions of one or more of the figures in this paper are available online at http://ieeexplore.ieee.org.

Digital Object Identifier 10.1109/JQE.2009.2028900 tuned far beyond the relaxation resonance frequency of the laser. The period-one oscillation can be optically controlled, easily locked, and deeply modulated. As a photonic microwave source, the laser in period-one oscillation has been applied for narrow-linewidth microwave signal generation, radio-over-fiber (RoF) subcarrier transmission, wavelength conversion, signal AM-to-FM conversion, and remote target detection [13]-[17].

Despite the many potential applications of period-one oscillation, most related studies have been conducted through numerical simulations only. The nonlinear nature of the laser system prohibits exact analytical investigations. There have been a few excellent reports that qualitatively explained the oscillation using perturbation analysis, multi-time scale analysis, or bifurcation analysis [18]-[23]. However, quantitative characterization of the period-one oscillation for microwave generation, to the best of our knowledge, has not been reported.

In this paper, analytical solution of the oscillation based on a two-wavelength approximation is reported for the first time. The laser emission is approximated to be comprised of two dominating wavelengths. We obtain theoretical results that relate the oscillation characteristics to the operating conditions, intrinsic laser parameters, and injection parameters. In particular, mathematical expressions are obtained for the microwave power, optical power, and injection strength, where the injection detuning frequency and the period-one oscillation frequency are treated as input variables.

Most importantly, the results explain the physical mechanism behind the period-one oscillation. The oscillation can be viewed as the beating of two dominating wavelengths. One is regenerated from the optical injection while the other is emitted near the cavity resonance wavelength. When the injection strength increases, the optical gain normally decreases due to saturation, the cavity resonance is then red-shifted through the antiguidance effect. As a result, the beat frequency increases continuously with the injection strength. The role of the cavity resonance shifting has been speculated and simulated previously [14], [24], [25], but it is confirmed and evaluated by our analysis for the first time. In fact, the mechanism is only approximately valid when the antiguidance factor is large and the gain compression factor is small.

The well-established rate-equation model is used throughout the analysis. The model has been proposed based on fundamental laser physics. Thus far, numerical simulations on the rate equations have consistently yielded excellent quantitative agreement with experiments, which have been conducted extensively over many years [1], [2], [6], [7], [9]. Building on these findings, numerical simulations are employed to verify our analytical results in the present paper. Nevertheless, all of our laser parameters were obtained experimentally. They were extracted from 
a 1.3- $\mu \mathrm{m}$ laser using a four-wave mixing parameter extraction technique [26], [27]. Therefore, we focus on establishing our theoretical results using the realistic simulation results as our reference. The results are in good agreement.

Following this introduction, the theoretical model is presented in Section II. Then, the two-wavelength approximation is presented in Section III. Analytical results are compared to numerical results in Section IV, which are then followed by discussions and conclusion in Sections V and VI, respectively.

\section{THEORETICAL MODEL}

Semiconductor lasers are dynamically class B lasers which do not require consideration of the polarization [3], [24]. The dynamical behavior is fully described by the temporal evolution of the complex optical field and the charge carrier density. So a single-mode semiconductor laser under constant, coherent optical injection is a three-dimensional system, which can be described by state variables $(A(t), N(t))$. Here, $A$ is the complex intracavity field amplitude in reference to the optical frequency of the injection and $N$ is the charge carrier density of the laser. For simplicity, the state variables are normalized to become $a(t)=A /\left|A_{0}\right|$ and $\tilde{n}(t)=\left(N-N_{0}\right) / N_{0}$, where $A_{0}$ and $N_{0}$ are the free-running values of $A$ and $N$, respectively. Note that $a$ is complex while $\tilde{n}$ is real.

The laser is controlled by the optical injection parameters $\left(\xi_{i}, \Omega_{i}\right)$, which denote the injection strength and the injection detuning frequency, respectively. The injection strength is defined as the injected field strength normalized to the emitted field strength of a free-running laser. The injection frequency detuning is defined as the offset frequency of the injection measured from the free-running frequency of the laser.

By normalizing the established model [14], [24], [26], the rate equations that govern $(a, \tilde{n})$ under optical injection of $\left(\xi_{\mathbf{i}}, \Omega_{\mathbf{i}}\right)$ are given by

$$
\begin{aligned}
& \frac{\mathrm{d} a}{\mathrm{~d} t}=\mathrm{i} \Omega_{\mathrm{i}} a+\frac{1-\mathrm{i} b}{2}\left(\Gamma \mathrm{g}-\gamma_{\mathrm{c}}\right) a+\gamma_{\mathrm{c}} \xi_{\mathrm{i}}, \\
& \frac{\mathrm{d} \tilde{n}}{\mathrm{~d} t}=\gamma_{\mathrm{s}} \tilde{J}-\gamma_{\mathrm{s}} \tilde{n}-\frac{\gamma_{\mathrm{s}} \tilde{J}}{\gamma_{\mathrm{c}}} \Gamma g|a|^{2}
\end{aligned}
$$

where the gain $g$ is given by

$$
\mathrm{g}=\Gamma^{-1}\left[\gamma_{\mathrm{c}}+\frac{\gamma_{\mathrm{c}} \gamma_{\mathrm{n}}}{\gamma_{\mathrm{s}} \tilde{J}} \tilde{n}-\gamma_{\mathrm{p}}\left(|a|^{2}-1\right)\right]
$$

In (1)-(3), $\gamma_{\mathrm{c}}$ is the cavity decay rate, $\gamma_{\mathrm{s}}$ is the spontaneous carrier relaxation rate, $\gamma_{\mathrm{n}}$ is the differential carrier relaxation rate, $\gamma_{\mathrm{p}}$ is the nonlinear carrier relaxation rate, $b$ is the antiguidance factor, $\Gamma$ is the confinement factor of the optical mode inside the gain medium, and $\tilde{J}$ is the normalized bias current above threshold. These dynamical parameters are fixed as long as the bias and the temperature are kept constant. They are independent of the injection parameters and the laser dynamics. The gain $g$ depends on both carrier and photon densities [14], [28]. The expression in (3) is obtained by noting that the free-running photon density equals $\Gamma \gamma_{\mathrm{s}} N_{0} \tilde{J} / \gamma_{\mathrm{c}}$. Detailed discussion on the dynamical parameters can be found in the literatures [26], [29].

The values of the parameters are summarized in Table I. The laser is biased at $\tilde{J}=1.222$ and emits about $4.5 \mathrm{~mW}$ of optical
TABLE I

SUMMARY OF SYMBOLS

\begin{tabular}{lll}
\hline \hline Parameter & Symbol & Remarks/Value \\
\hline Normalized complex optical field & $a$ & Equation 1 \\
Normalized carrier density & $\tilde{n}$ & Equation 2 \\
Cavity decay rate & $\gamma_{\mathrm{c}}$ & $5.36 \times 10^{11} \mathrm{~s}^{-1}$ \\
Spontaneous carrier relaxation rate & $\gamma_{\mathrm{s}}$ & $5.96 \times 10^{9} \mathrm{~s}^{-1}$ \\
Differential carrier relaxation rate & $\gamma_{\mathrm{n}}$ & $7.53 \times 10^{9} \mathrm{~s}^{-1}$ \\
Nonlinear carrier relaxation rate & $\gamma_{\mathrm{p}}$ & $1.91 \times 10^{10} \mathrm{~s}^{-1}$ \\
Antiguidance factor & $b$ & 3.2 \\
Normalized current above threshold & $\tilde{J}$ & 1.222 \\
Relaxation resonance frequency & $\Omega_{\mathrm{r}}$ & $2 \pi \times 10.25 \mathrm{GHz}$
\end{tabular}

Injection parameters

Injection strength

Injection detuning frequency

$\xi_{\mathrm{i}} \quad$ Equation 1

Period-one oscillation parameters

Period-one oscillation frequency

Cavity resonance frequency shift

Regenerated optical field

Generated optical field

Average output intensity

Output intensity oscillation amplitude

intensity oscillation amplitude $s_{1}$

Carrier density oscillation amplitude $\tilde{n}_{1}$

Combined relaxation rate

$\Omega_{\mathrm{i}} \quad$ Equation 1

power. The relaxation resonance frequency is $\Omega_{\mathrm{r}}=\left(\gamma_{\mathrm{c}} \gamma_{\mathrm{n}}+\right.$ $\left.\gamma_{\mathrm{s}} \gamma_{\mathrm{p}}\right)^{1 / 2}$, which is equal to $2 \pi \times 10.25 \mathrm{GHz}$. It should be mentioned that, without optical injection, the laser cannot be directly modulated much beyond $\Omega_{\mathrm{r}}$ using conventional methods. However, with optical injection, the laser can be driven into period-one oscillation at frequencies several times higher than $\Omega_{\mathrm{r}}$.

Throughout this paper, both theoretical analysis and numerical simulation are based on the above rate-equation model in (1)-(3). All simulation results are obtained from the secondorder Runge-Kutta numerical integration method with time step and time span of 238 fs and 125 ns, respectively. Besides, according to (3), $\gamma_{p}$ represents the gain compression effect of the laser. While the complete model with $\gamma_{p}$ is more consistent with experiments, it is sometimes ignored in related studies by setting $\gamma_{\mathrm{p}}=0$ [30], [31]. For completeness, both cases with and without $\gamma_{\mathrm{p}}$ will be examined in the rest of the paper.

Different laser dynamics are observed over the injection parameter space $\left(\xi_{\mathrm{i}}, \Omega_{\mathrm{i}}\right)$. Through comprehensive simulations, Figs. 1(a) and (b) show various dynamic regions for $\gamma_{p}=0$ and $1.91 \times 10^{10} \mathrm{~s}^{-1}$, respectively. The figures are mappings of the sideband suppression ratio $R$, which will be discussed in the next section. At this point, it suffices to observe the clear boundaries between different dynamic regions. These boundaries can also be determined by bifurcation analysis and small-signal analysis [7], [24]. In each map, there are small regions of complicated nonlinear dynamics including period-two oscillation, high-order periodic, quasi-periodic, chaos, and unlocked dynamics. There is also a region of stable locking that is bounded from above by a Hopf bifurcation line. When $\Omega_{\mathrm{i}}$ is increased above the Hopf bifurcation line, a large region of period-one oscillation is found. The laser exhibits single-period oscillation at a tunable frequency $\Omega_{0}$. The corresponding intensity oscillation can be converted into microwave signal using a high-speed photodetector. Thus, it is important to analyze the characteristics of the period-one oscillations. 

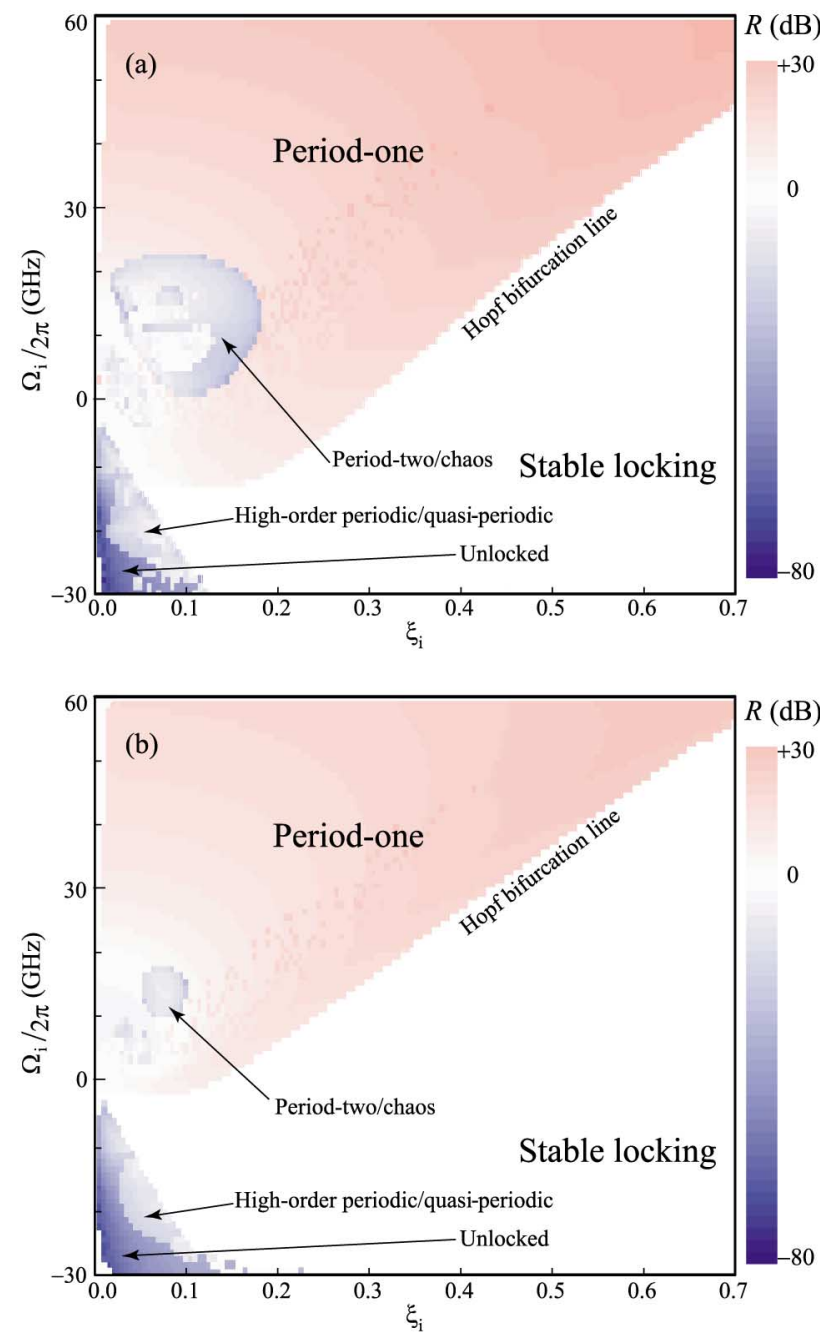

Fig. 1. Mappings of dynamic regions of the optically injected semiconductor laser with (a) $\gamma_{\mathrm{p}}=0$ and (b) $\gamma_{\mathrm{p}}=1.91 \times 10^{10} \mathrm{~s}^{-1}$. The sideband suppression ratio $R$ is presented in color.

As an example of period-one oscillation, a simulated optical spectrum is shown as the solid curve in Fig. 2. The optical frequency axis is offset to the free-running frequency of the laser. The injection parameters are set as $\left(\xi_{\mathrm{i}}, \Omega_{\mathrm{i}}\right)=(0.32,2 \pi \times$ $30 \mathrm{GHz})$. The spectrum consists of the regenerative component at $\Omega_{\mathrm{i}}$ and sidebands equally separated by $\Omega_{0}$, where $\Omega_{0}=$ $2 \pi \times 38 \mathrm{GHz}$ in this case. Additionally, the dashed curve of Fig. 2 shows the spectrum when we set $\gamma_{p}=0$, while keeping everything else unchanged. Comparing the two spectra reveals that $\gamma_{p}$ does not have significant impact on the oscillation.

\section{Two-WAVELENGTH APPROXIMATION}

Under period-one oscillation, the state variables $(a, \tilde{n})$ can be expressed in terms of Fourier components at multiples of the oscillation frequency $\Omega_{0}$. Due to the laser nonlinearities, it is difficult to solve for all the components. We seek an approximate solution to the problem by retaining only the strongest of these components. According to Fig. 2, the strongest optical components for $a$ are at offset frequencies $\Omega_{\mathrm{i}}$ and $\Omega_{\mathrm{i}}-\Omega_{0}$. It is because the former is the direct regeneration of the injection while the latter is closest to the original cavity resonance

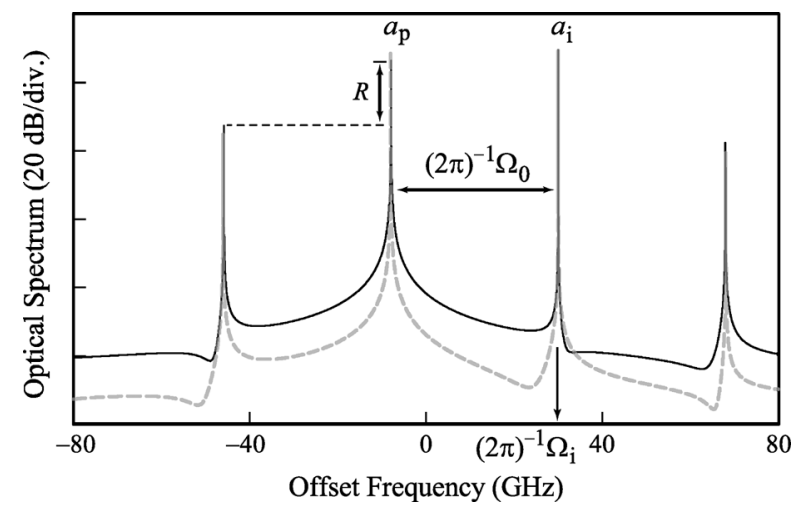

Fig. 2. Simulated optical spectrum of a period-one oscillation state at $\xi_{\mathrm{i}}=$ 0.32 and $\Omega_{\mathrm{i}}=2 \pi \times 30 \mathrm{GHz}$. The generated period-one oscillation frequency is $\Omega_{0}=2 \pi \times 38 \mathrm{GHz}$. Solid and dashed curves are obtained with and without $\gamma_{\mathrm{p}}$, respectively.

at the zero offset frequency. In fact, these two components consistently dominate the optical spectrum even when the injection parameters are changed.

In order to quantify such observation, we define the sideband suppression ratio $R$ by treating the laser as a two-wavelength light source. The weaker of the two dominating components is first chosen. It is then compared with the strongest component among the rest of the sidebands. The power difference in decibel is defined as $R$. For instance, in the spectrum shown as the solid curve in Fig. 2, the component at $\Omega_{\mathrm{i}}-\Omega_{0}$ is slightly weaker than that at $\Omega_{\mathrm{i}}$. It is thus compared with the strongest sideband, which turns out to be located at $\Omega_{\mathrm{i}}-2 \Omega_{0}$. The sideband suppression ratio is thus determined as $R=20 \mathrm{~dB}$. Mappings of $R$ over the injection parameter space are shown in Fig. 1. Abrupt changes occur at the boundaries between different dynamics because of sudden changes in the oscillation frequency and the spectrum. Within the period-one oscillation region, $R$ gradually increases with both $\xi_{\mathrm{i}}$ and $\Omega_{\mathrm{i}}$. Comparing the two mappings reveals that the gain compression effect of $\gamma_{\mathrm{p}}$ tends to reduce $R$. For the cases with and without $\gamma_{\mathrm{p}}, R$ is always greater than 10 $\mathrm{dB}$ when $(2 \pi)^{-1} \Omega_{\mathrm{i}}$ is above 31 and $0 \mathrm{GHz}$, respectively. Therefore, it is reasonable to keep only the strongest components $\Omega_{\mathrm{i}}$ and $\Omega_{\mathrm{i}}-\Omega_{0}$ over a large range of injection conditions. Such two-wavelength approximation will be used throughout the theoretical analysis. It should be pointed out that as $R$ is reduced by decreasing $\Omega_{\mathrm{i}}$, the two-wavelength approximation and the performance of the analysis degrade. For example, the analysis does not yield good results for our laser when $\Omega_{\mathrm{i}}<0$ for $\gamma_{p}=0$. Extension of the analysis beyond the two-wavelength approximation is beyond the current scope.

Applying the two-wavelength approximation, the field $a$ is written as

$$
a \approx a_{\mathrm{p}} \mathrm{e}^{\mathrm{i} \Omega_{0} t}+a_{\mathrm{i}}
$$

where $a_{\mathrm{i}}$ is regenerated from the injection and $a_{\mathrm{p}}$ is generated by the laser in period-one oscillation (Fig. 2). It follows that the output intensity of the laser, after normalizing to the freerunning intensity, is given by

$$
|a|^{2}=s_{0}+\left(s_{1} \mathrm{e}^{-\mathrm{i} \Omega_{0} t}+\text { c.c. }\right)
$$


where $s_{0}=\left|a_{\mathrm{p}}\right|^{2}+\left|a_{\mathrm{i}}\right|^{2}$ is the time-averaged intensity and $s_{1}=a_{\mathrm{p}}^{*} a_{\mathrm{i}}$ is the complex amplitude of the intensity oscillation at $\Omega_{0}$. Here, c.c. stands for complex conjugate. Similarly, since the oscillation frequency $\Omega_{0}$ is typically much higher than the spontaneous carrier relaxation rate $\gamma_{\mathrm{s}}$

$$
\tilde{n} \approx \tilde{n}_{0}+\left(\tilde{n}_{1} \mathrm{e}^{-\mathrm{i} \Omega_{0} t}+\text { c.c. }\right)
$$

where $\tilde{n}_{0}$ is the time-averaged carrier density and $\tilde{n}_{1}$ is the oscillation amplitude of the carrier density at the period-one frequency. The harmonics of the oscillation are neglected.

The approximations in (4)-(6) will be adopted throughout the analysis. The analysis is based on the rate equations (1) and (2). They are rewritten as

$$
\begin{aligned}
-\mathrm{i} \Omega_{\mathrm{i}} a_{\mathrm{i}}= & \frac{1-\mathrm{i} b}{2}\left(\frac{\gamma_{\mathrm{c}} \gamma_{n}}{\gamma_{\mathrm{s}} \tilde{J}} \tilde{n}_{1} a_{\mathrm{p}}+\frac{2 \Omega_{\mathrm{s}}}{b} a_{\mathrm{i}}-\gamma_{\mathrm{p}}\left|a_{\mathrm{p}}\right|^{2} a_{\mathrm{i}}\right) \\
& +\gamma_{\mathrm{c}} \xi_{\mathrm{i}} \\
\mathrm{i}\left(\Omega_{0}-\Omega_{\mathrm{i}}\right) a_{\mathrm{p}}= & \frac{1-\mathrm{i} b}{2} \\
& \times\left(\frac{\gamma_{\mathrm{c}} \gamma_{n}}{\gamma_{\mathrm{s}} \tilde{J}} \tilde{n}_{1}^{*} a_{\mathrm{i}}+\frac{2 \Omega_{\mathrm{s}}}{b} a_{\mathrm{p}}-\gamma_{\mathrm{p}}\left|a_{\mathrm{i}}\right|^{2} a_{\mathrm{p}}\right) \\
\gamma \tilde{n}_{0}= & -\gamma_{\mathrm{s}} \tilde{J}\left[s_{0}-1-\frac{\gamma_{\mathrm{p}}}{\gamma_{\mathrm{c}}}\left(s_{0}\left(s_{0}-1\right)+2\left|s_{1}\right|^{2}\right)\right] \\
& -\gamma_{\mathrm{n}}\left(\tilde{n}_{1}^{*} s_{1}+\text { c.c. }\right) \\
\left(\mathrm{i} \Omega_{0}-\gamma\right) \tilde{n}_{1}= & \gamma_{\mathrm{s}} \tilde{J} s_{1}\left(1+\frac{2 \Omega_{\mathrm{s}}}{b \gamma_{\mathrm{c}}}-\frac{\gamma_{\mathrm{p}}}{\gamma_{c}} s_{0}\right)
\end{aligned}
$$

where

$$
\gamma=\gamma_{\mathrm{s}}+\gamma_{\mathrm{n}} s_{0}
$$

and

$$
\begin{aligned}
\Omega_{\mathrm{s}} & =b \frac{\Gamma}{2}\left\langle\mathrm{~g}-\frac{\gamma_{\mathrm{c}}}{\Gamma}\right\rangle \\
& =\frac{b}{2}\left[\frac{\gamma_{\mathrm{c}} \gamma_{\mathrm{n}}}{\gamma_{\mathrm{s}} \tilde{J}} \tilde{n}_{0}-\gamma_{\mathrm{p}}\left(s_{0}-1\right)\right]
\end{aligned}
$$

Inspecting (12) and (1) reveals that $\Omega_{\mathrm{S}}$ is a very important physical quantity. It is the shift of the cavity resonance frequency under the influence of optical injection. Usually, optical injection reduces the charge carrier density of the cavity. The timeaveraged gain $\langle\mathrm{g}\rangle$ thus reduces from its free-running value $\gamma_{c} / \Gamma$. The reduction of gain induces, through the antiguidance factor $b$, an increase of the refractive index. The corresponding effective cavity length increases and so the cavity resonance frequency is shifted by $\Omega_{\mathrm{s}}$. The frequency shift usually becomes more and more negative as the injection strength increases. The frequency plays an important role in the tunability of the period-one oscillation frequency, as the next section will show.

\section{RESULTS}

In this section, the period-one oscillation characteristics are derived using the simplified rate equations (7)-(10). For any given injection detuning frequency $\Omega_{\mathrm{i}}$, the generated period-one oscillation frequency $\Omega_{0}$ can be tuned by varying the injection
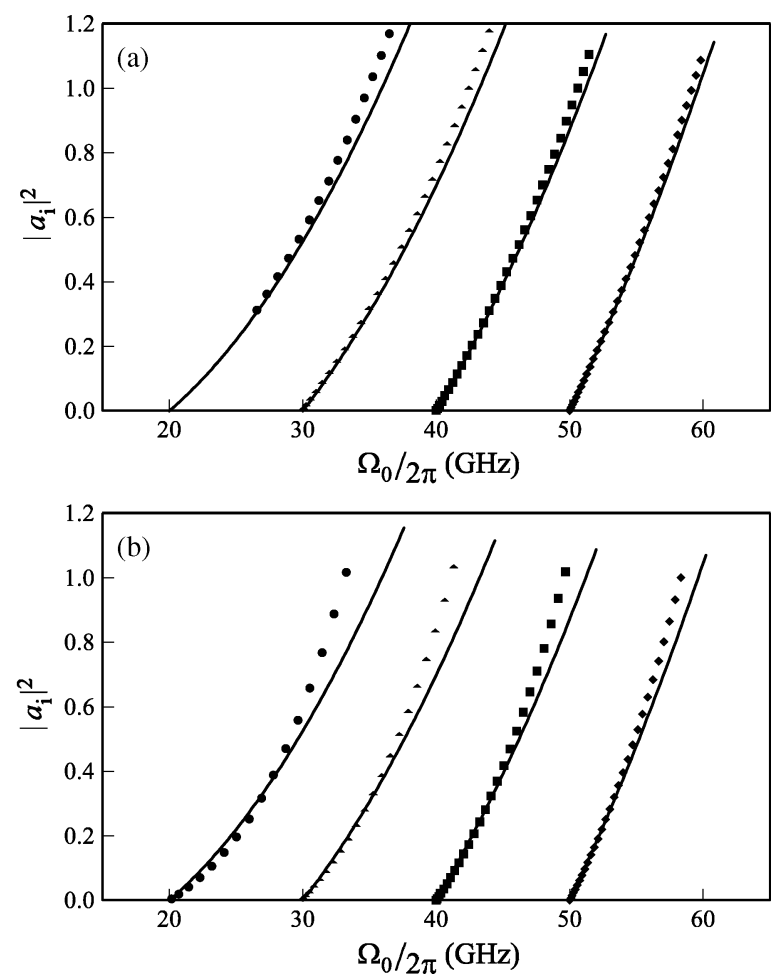

Fig. 3. Regenerative optical component $\left|a_{\mathrm{i}}\right|^{2}$ as a function of the period-one oscillation frequency $\Omega_{0}$. The solid curves represent analytical results while the closed symbols represent numerical results, where $(2 \pi)^{-1} \Omega_{\mathrm{i}}=20 \mathrm{GHz}$ (circles), $30 \mathrm{GHz}$ (triangles), $40 \mathrm{GHz}$ (squares), and $50 \mathrm{GHz}$ (diamonds). (a) $\gamma_{\mathrm{p}}=$ 0. (b) $\gamma_{\mathrm{p}}=1.91 \times 10^{10} \mathrm{~s}^{-1}$.

strength $\xi_{\mathrm{i}}$. Thus, both $\Omega_{\mathrm{i}}$ and $\Omega_{0}$ are treated as input variables in the following derivations of the other quantities, which include the regenerative optical component $\left|a_{\mathrm{i}}\right|^{2}$, the cavity resonance frequency shift $\Omega_{\mathrm{s}}$, the average output intensity $s_{0}$, the generated period-one optical component $\left|a_{\mathrm{p}}\right|^{2}$, the generated microwave power $\left|s_{1}\right|^{2}$, and the required injection strength $\xi_{\mathrm{i}}$.

The resultant analytical expressions are verified through extensive numerical simulations. The results are presented in Figs. 3-8. The solid curves are from analytical results while the closed symbols are from numerical results. The analytical results are obtained from directly evaluating the expressions to be presented in this section. The numerical results are obtained from the numerical integration presented in Section II. In each figure, the results are shown for $(2 \pi)^{-1} \Omega_{\mathrm{i}}=20,30,40$, and $50 \mathrm{GHz}$, which are marked by circles, triangles, squares, and diamonds, respectively. For completeness, both cases with $\gamma_{\mathrm{p}}=0$ and $1.91 \times 10^{10} \mathrm{~s}^{-1}$ are shown in every part (a) and part (b), respectively.

\section{A. Cavity Shift}

By combining (8) and (10), we can eliminate $\tilde{n}_{1}$ using $s_{1}=$ $a_{\mathrm{p}}^{*} a_{\mathrm{i}}$ to yield

$$
\begin{aligned}
{\left[-\mathrm{i}\left(\Omega_{0}-\Omega_{\mathrm{i}}\right)+\right.} & \left.\frac{1-\mathrm{i} b}{2}\left(\frac{2 \Omega_{\mathrm{s}}}{b}-\gamma_{\mathrm{p}}\left|a_{\mathrm{i}}\right|^{2}\right)\right]\left(\mathrm{i} \Omega_{0}+\gamma\right) \\
& =\frac{1-\mathrm{i} b}{2} \gamma_{\mathrm{n}}\left(\gamma_{\mathrm{c}}+\frac{2 \Omega_{\mathrm{s}}}{b}-\gamma_{\mathrm{p}} s_{0}\right)\left|a_{\mathrm{i}}\right|^{2}
\end{aligned}
$$



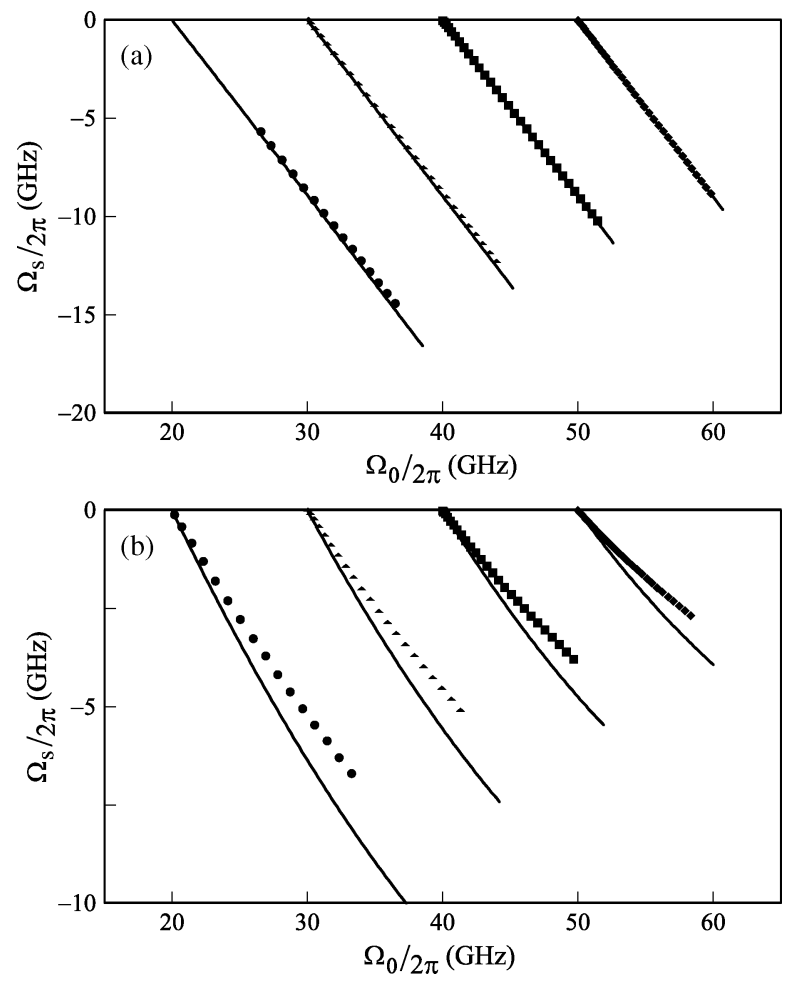

Fig. 4. Cavity resonance frequency shift $\Omega_{\mathrm{s}}$ as a function of the period-one oscillation frequency $\Omega_{0}$. The solid curves represent analytical results while the closed symbols represent numerical results, where $(2 \pi)^{-1} \Omega_{\mathrm{i}}=20 \mathrm{GHz}$ (circles), $30 \mathrm{GHz}$ (triangles), $40 \mathrm{GHz}$ (squares), and $50 \mathrm{GHz}$ (diamonds). (a) $\gamma_{\mathrm{p}}=$ 0. (b) $\gamma_{\mathrm{p}}=1.91 \times 10^{10} \mathrm{~s}^{-1}$.

for $a_{\mathrm{p}} \neq 0$. Solving the real and imaginary parts, we obtain

$$
\left|a_{\mathrm{i}}\right|^{2}=\frac{2\left(\Omega_{0}^{2}+\gamma^{2}\right)}{\gamma_{\mathrm{n}}\left(\gamma_{\mathrm{c}}+\frac{2 \Omega_{\mathrm{s}}}{b}-\gamma_{\mathrm{p}} s_{0}\right)\left(1+b^{2}\right) \Omega_{0}}\left(\Omega_{0}-\Omega_{\mathrm{i}}\right)
$$

and

$$
\Omega_{\mathrm{s}}=-\frac{\left(b \Omega_{0}-\gamma\right)\left(\Omega_{0}-\Omega_{\mathrm{i}}\right)}{\left(1+b^{-2}\right) b \Omega_{0}}+\frac{b \gamma_{\mathrm{p}}}{2}\left|a_{\mathrm{i}}\right|^{2} .
$$

Both $\left|a_{\mathrm{i}}\right|^{2}$ and $\Omega_{\mathrm{s}}$ depend strongly on $\left(\Omega_{0}, \Omega_{\mathrm{i}}\right)$. Some additional approximations are helpful in simplifying the results. For most semiconductor lasers, $b \sim 2-9, \gamma_{\mathrm{p}} \sim 10^{10} \mathrm{~s}^{-1}$, and $s_{0} \sim 1$ even under injection. Also, $\gamma_{\mathrm{c}} \sim 10^{11}-10^{12} \mathrm{~s}^{-1}$ is always the fastest rate among the parameters in the model and is much higher than the magnitude of the cavity resonance frequency shift $\Omega_{\mathrm{s}}$. As a result, we assume that (i) $\gamma_{\mathrm{c}} \gg\left|\left(2 \Omega_{\mathrm{s}} / b\right)-\gamma_{\mathrm{p}} s_{0}\right|$ and (ii) $\gamma \approx \gamma_{\mathrm{s}}+\gamma_{\mathrm{n}}$. These are the only extra assumptions applied in the analysis other than the two-wavelength approximation. They allow evaluation of $\left|a_{\mathrm{i}}\right|^{2}$ and $\Omega_{\mathrm{s}}$ as functions of $\left(\Omega_{0}, \Omega_{\mathrm{i}}\right)$ using (15) and (16), respectively.

Fig. 3 shows the regenerative optical component $\left|a_{\mathrm{i}}\right|^{2}$ as a function of $\Omega_{0}$ under different $\Omega_{\mathrm{i}}$. The analytical results in solid curves agree well with the numerical results in closed symbols. Each curve follows a similar trend. When $\Omega_{0}$ is equal to $\Omega_{\mathrm{i}}$, the laser must be under simple four-wave mixing, where the laser is just slightly perturbed by the injection. This occurs if the injection strength is infinitesimally small and so $\left|a_{\mathrm{i}}\right|^{2}$ is zero.
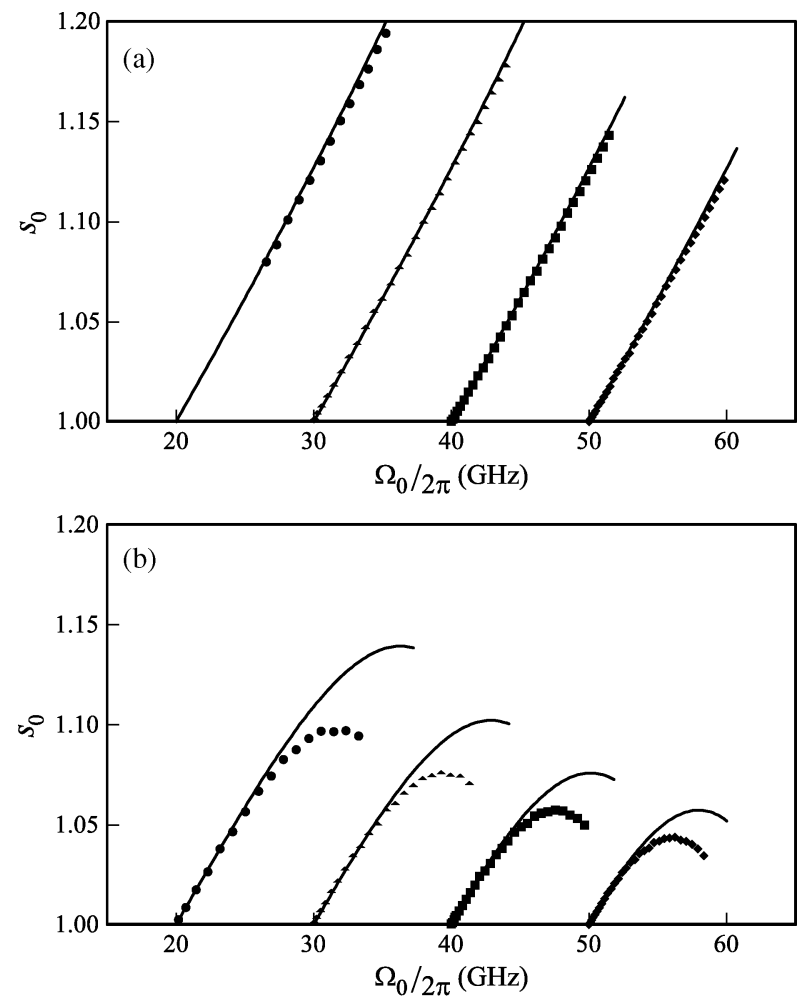

Fig. 5. Average optical output intensity $s_{0}$ as a function of the period-one oscillation frequency $\Omega_{0}$. The solid curves represent analytical results while the closed symbols represent numerical results, where $(2 \pi)^{-1} \Omega_{\mathrm{i}}=20 \mathrm{GHz}$ (circles), $30 \mathrm{GHz}$ (triangles), $40 \mathrm{GHz}$ (squares), and $50 \mathrm{GHz}$ (diamonds). (a) $\gamma_{\mathrm{p}}=0$. (b) $\gamma_{\mathrm{p}}=1.91 \times 10^{10} \mathrm{~s}^{-1}$.
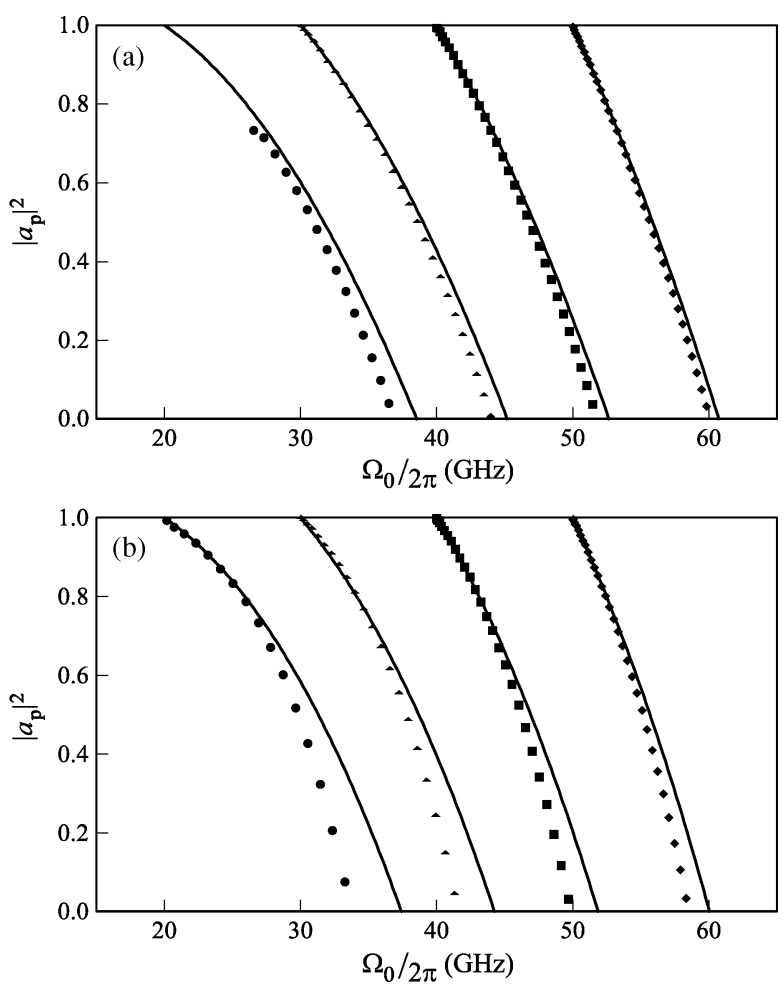

Fig. 6. Generated optical component $\left|a_{\mathrm{p}}\right|^{2}$ as a function of the period-one oscillation frequency $\Omega_{0}$. The solid curves represent analytical results while the closed symbols represent numerical results, where $(2 \pi)^{-1} \Omega_{\mathrm{i}}=20 \mathrm{GHz}$ (circles), $30 \mathrm{GHz}$ (triangles), $40 \mathrm{GHz}$ (squares), and $50 \mathrm{GHz}$ (diamonds). (a) $\gamma_{\mathrm{p}}=$ 0 . (b) $\gamma_{\mathrm{p}}=1.91 \times 10^{10} \mathrm{~s}^{-1}$. 

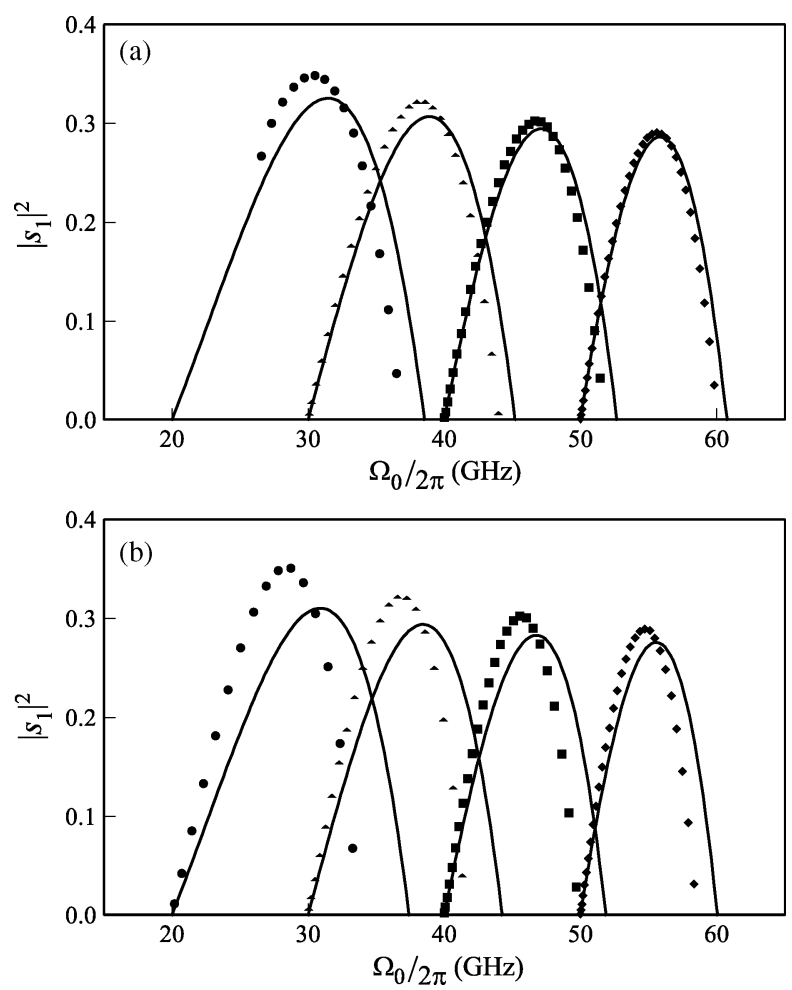

Fig. 7. Microwave power $\left|s_{1}\right|^{2}$ as a function of the period-one oscillation frequency $\Omega_{0}$. The solid curves represent analytical results while the closed symbols represent numerical results, where $(2 \pi)^{-1} \Omega_{\mathrm{i}}=20 \mathrm{GHz}$ (circles), $30 \mathrm{GHz}$ (triangles), $40 \mathrm{GHz}$ (squares), and $50 \mathrm{GHz}$ (diamonds). (a) $\gamma_{\mathrm{p}}=0$. (b) $\gamma_{\mathrm{p}}=$ $1.91 \times 10^{10} \mathrm{~s}^{-1}$.
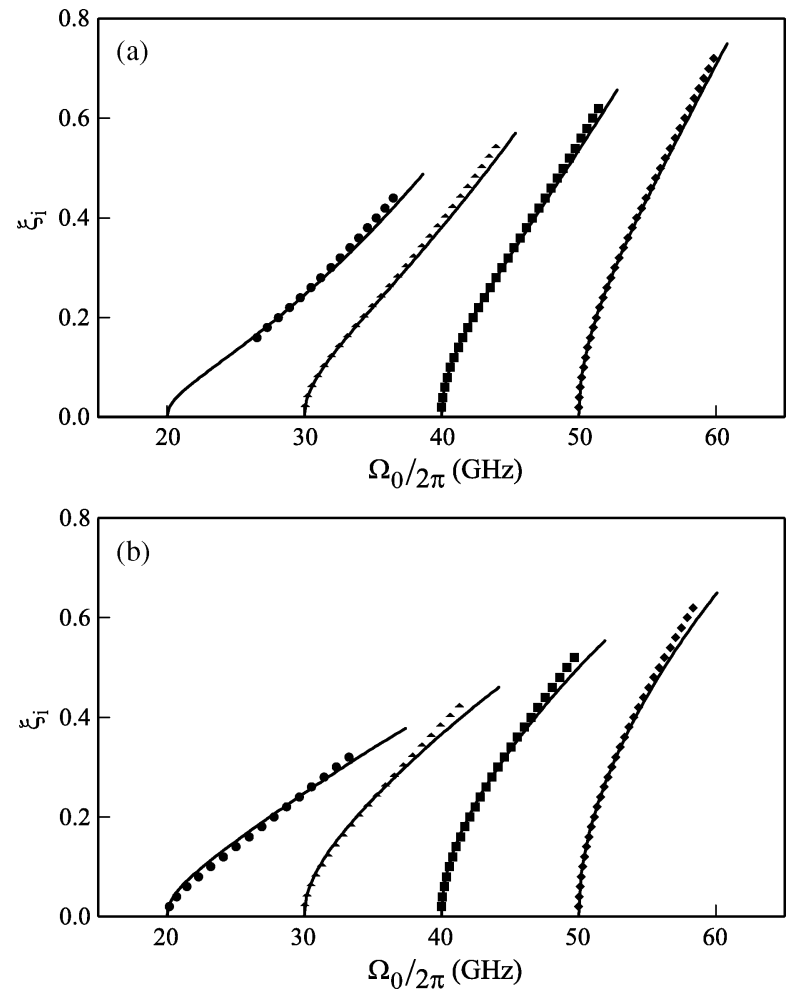

Fig. 8. Required optical injection strength $\xi_{\mathrm{i}}$ as a function of the period-one oscillation frequency $\Omega_{0}$. The solid curves represent analytical results while the closed symbols represent numerical results, where $(2 \pi)^{-1} \Omega_{\mathrm{i}}=20 \mathrm{GHz}$ (circles), $30 \mathrm{GHz}$ (triangles), $40 \mathrm{GHz}$ (squares), and $50 \mathrm{GHz}$ (diamonds). (a) $\gamma_{\mathrm{p}}=$ 0. (b) $\gamma_{\mathrm{p}}=1.91 \times 10^{10} \mathrm{~s}^{-1}$.
However, as $\Omega_{0}$ increases, the injection strength has to be increased accordingly. This leads to an increase of $\left|a_{\mathrm{i}}\right|^{2}$ through regeneration. As a side note, for the simulation data in Fig. 3(a) at $\Omega_{\mathrm{i}}=2 \pi \times 20 \mathrm{GHz}$, the minimum $\Omega_{0}$ is $2 \pi \times 26 \mathrm{GHz}$ at $\xi_{\mathrm{i}}=0.15$. The frequency $\Omega_{0}$ cannot be decreased further by reducing the injection strength $\xi_{\mathrm{i}}$. According to the mapping in Fig. 1(a), the laser exhibits period-two and chaotic oscillations for small $\xi_{\mathrm{i}}$ when $\Omega_{\mathrm{i}}=2 \pi \times 20 \mathrm{GHz}$. Our analysis is only applicable to period-one oscillations.

Fig. 4 shows the cavity resonance frequency shift $\Omega_{\mathrm{s}}$ obtained as $\Omega_{0}$ varies. The analytical results are calculated by substituting (15) into (16). For any given $\Omega_{\mathrm{i}}$, increasing $\Omega_{0}$ requires $\xi_{\mathrm{i}}$ to increase accordingly. The cavity resonance frequency shift $\Omega_{\mathrm{S}}$ becomes more and more negative because of the physical argument presented in Section III. Contrasting Figs. 4(a) and (b), the agreement between analytical and numerical results is better if $\gamma_{\mathrm{p}}$ can be ignored. This is because the two-wavelength approximation is more plausible when there is no gain compression.

In order to obtain some physical insight, the case with $\gamma_{\mathrm{p}}=0$ is considered here. Typically, $b \Omega_{0} \gg \gamma$ and $b^{-2} \ll 1$ so that (16) can be simplified to

$$
\Omega_{\mathrm{s}} \approx \Omega_{\mathrm{i}}-\Omega_{0} .
$$

This implies that the period-one oscillation is caused by beating of light from the injection at $\Omega_{\mathrm{i}}$ and the emission at the shifted cavity resonance frequency $\Omega_{\mathrm{s}}$. In other words, the period-one oscillation is interpreted as a generalized four-wave mixing with the cavity resonance frequency being shifted by strong injection. Such mechanism has been proposed by the pioneers of this subject [32]. The role of $\Omega_{\mathrm{s}}$ has been speculated for the stable locking state as well [25], [31]. However, it is confirmed analytically for the first time as a result of the two-wavelength approximation. The situation becomes more complicated if $\gamma_{p}$ cannot be neglected.

\section{B. Averaged Intensity}

Substituting (16) into (8), the following can be obtained

$$
\tilde{n}_{1}^{*} s_{1}=\frac{2\left(\mathrm{i} \Omega_{0}-\gamma\right)}{1+b^{2}} \frac{\gamma_{\mathrm{s}} \tilde{J}}{\gamma_{\mathrm{c}} \gamma_{\mathrm{n}}} \frac{\Omega_{0}-\Omega_{\mathrm{i}}}{\Omega_{0}}\left|a_{\mathrm{p}}\right|^{2} .
$$

Putting (18) into (9) yields the normalized average optical output intensity

$$
s_{0}=1+2 \frac{-\left(\gamma_{\mathrm{s}}+\gamma_{\mathrm{n}}\right) \Omega_{\mathrm{s}}+2 \gamma_{\mathrm{n}} \delta\left(1-\left|a_{\mathrm{i}}\right|^{2}\right)}{b \Omega_{\mathrm{r}}^{2}+2 \gamma_{\mathrm{n}} \Omega_{\mathrm{s}}-4 \gamma_{\mathrm{n}} \delta}
$$

where we define $\delta$ for convenience as

$$
\delta=\Omega_{\mathrm{s}}+\frac{\Omega_{0}-\Omega_{\mathrm{i}}}{1+b^{-2}} .
$$

Fig. 5 shows the results for $s_{0}$. Every curve starts with $\Omega_{0}=\Omega_{\mathrm{i}}$ when the injection is infinitesimally small. The emitted intensity $s_{0}$ equals unity, as for a free-running laser. When $\Omega_{0}$ increases, the required $\xi_{\mathrm{i}}$ increases, so $s_{0}$ increases accordingly. Analytical and numerical results are in excellent agreement when $\gamma_{\mathrm{p}}=0$ in Fig. 5(a). In Fig. 5(b), the model also predicts the decrease of slopes at high $s_{0}$ due to the saturation effect of a non-zero $\gamma_{\mathrm{p}}$. The agreement between analytical and simulation results is 
again better for $\gamma_{\mathrm{p}}=0$ because of the two-wavelength approximation.

\section{Period-One Components}

The optical spectral component generated by the period-one oscillation can be evaluated as

$$
\left|a_{\mathrm{p}}\right|^{2}=s_{0}-\left|a_{\mathrm{i}}\right|^{2}
$$

where (15) and (19) are used. The results are plotted in Fig. 6. When $\Omega_{0}=\Omega_{\mathrm{i}}$, the injection is very weak, $a_{\mathrm{i}}=0$ and $s_{0}=$ 1 , so that $\left|a_{\mathrm{p}}\right|^{2}=1$. When $\Omega_{0}$ increases, $\left|a_{\mathrm{i}}\right|^{2}$ grows and $\Omega_{\mathrm{s}}$ becomes increasingly negative. Eventually, $\left|a_{\mathrm{p}}\right|^{2}$ is reduced to zero as the laser undergoes a reverse Hopf bifurcation into stable locking.

Furthermore, the optical components $a_{\mathrm{p}}$ and $a_{\mathrm{i}}$ can beat at a photodetector. This yields a continuous-wave microwave signal at $\Omega_{0}$ with electrical power proportional to $\left|s_{1}\right|^{2}=\left|a_{\mathrm{p}} a_{\mathrm{i}}\right|^{2}$. By combining (15) and (21), $\left|s_{1}\right|^{2}$ is shown as a function of $\Omega_{0}$ in Fig. 7. There is always a peak for each curve because, from Figs. 3 and $6,\left|a_{\mathrm{i}}\right|^{2}$ and $\left|a_{\mathrm{p}}\right|^{2}$ have opposite dependencies on $\Omega_{0}$. The agreement between analytical and numerical results improves as $\Omega_{\mathrm{i}}$ increases. In relating to experiments, the photodetector is assumed to be $50-\Omega$ terminated and possess $0.5 \mathrm{~A} / \mathrm{W}$ responsivity. Since the free-running optical power of our laser is $4.5 \mathrm{~mW}$, we deduce that $\left|s_{1}\right|^{2}=0.1$ corresponds to microwave power of $-13 \mathrm{dBm}$. A transimpedance amplifier can be employed, as in most microwave photonics systems, if high-power microwave signal transmission is required.

\section{Tuning Characteristics}

The analysis finally relates the injection strength $\xi_{\mathrm{i}}$ to $\Omega_{0}$ by combining all earlier results. Putting (15) and (18) into (7), we have

$$
\begin{aligned}
\xi_{\mathrm{i}}=\{[- & \left.\frac{\Omega_{\mathrm{s}}}{b}-\mathrm{i}\left(\Omega_{\mathrm{i}}-\Omega_{\mathrm{s}}\right)\right]- \\
& \left.\frac{1-\mathrm{i} b}{2}\left(\frac{\Omega_{\mathrm{r}}^{2}+\frac{2 \Omega_{s}}{b} \gamma_{\mathrm{n}}-\mathrm{i} \Omega_{0} \gamma_{\mathrm{p}}}{\mathrm{i} \Omega_{0}-\gamma}\right)\left|a_{\mathrm{p}}\right|^{2}\right\} \frac{a_{\mathrm{i}}}{\gamma_{\mathrm{c}}} .
\end{aligned}
$$

Since $\xi_{\mathrm{i}}$ is a positive real number, the phase of $a_{\mathrm{i}}$ can be deduced from (22). However, we only consider the magnitude of (22) in the following. By using the known results of $\left|a_{\mathrm{i}}\right|, \Omega_{\mathrm{s}}$, $s_{0}$, and $\left|a_{\mathrm{p}}\right|$, we obtain the required $\xi_{\mathrm{i}}$ as a function of the intended $\Omega_{0}$ under different $\Omega_{\mathrm{i}}$. The results are shown in Fig. 8, where excellent agreement between the analytical and numerical results is again observed. Since $\left(\xi_{\mathrm{i}}, \Omega_{\mathrm{i}}\right)$ are controlled by experimental settings, Fig. 8 can be regarded as the tuning curves of $\Omega_{0}$. The results are practically important in designing some RoF systems. For instance, they are applicable to understanding AM-to-FM converters, where the slopes and curvatures of Fig. 8 correspond to the conversion ratio and signal distortion, respectively. Hence, the two-wavelength analysis not only explains the physical mechanism of period-one oscillation but also provides guidance for designing microwave photonics systems.

\section{DISCUSSION}

The analysis on the period-one oscillation bridges the results previously known for four-wave mixing and stable locking. On

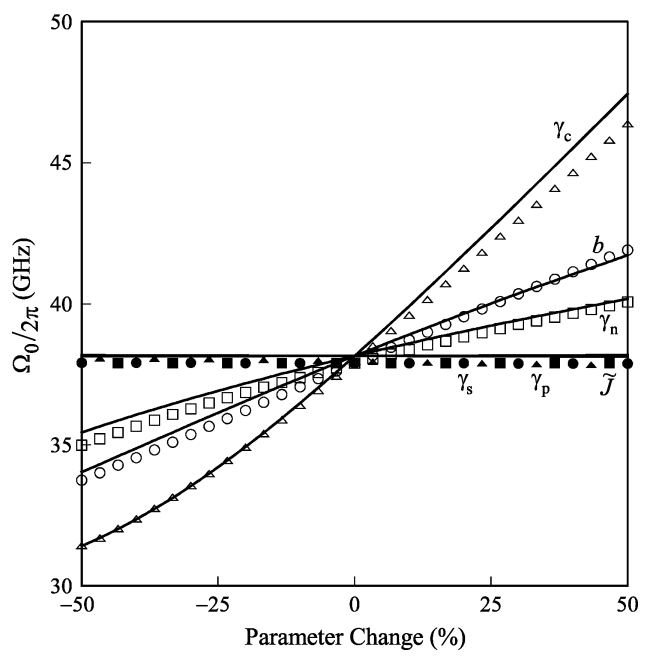

Fig. 9. Effects of the parameter changes on the period-one oscillation frequency $\Omega_{0}$. The solid curves represent analytical results while the symbols represent numerical results, where changes are made on $b$ (open circles), $\gamma_{c}$ (open triangles), $\gamma_{\mathrm{n}}$ (open squares), $\gamma_{\mathrm{s}}$ (closed circles), $\gamma_{\mathrm{p}}$ (closed triangles), and $\tilde{J}$ (closed squares).

one hand, four-wave mixing refers to the asymptotic behavior under very weak injection at large injection detuning frequency. Under four-wave mixing, $\Omega_{\mathrm{i}}-\Omega_{0} \approx \Omega_{\mathrm{s}} \approx 0$ and $\left|a_{\mathrm{p}}\right| \approx 1$. From the mappings in Fig. 1 , as $\Omega_{\mathrm{i}}$ increases along $\xi_{\mathrm{i}}=0$, the ratio $R$ increases and so the accuracy of the two-wavelength approximation improves. Considering $\Omega_{\mathrm{i}} \rightarrow \infty$, (22) is simplified to

$$
a_{\mathrm{i}}=\mathrm{i} \frac{\gamma_{\mathrm{c}} \xi_{\mathrm{i}}}{\Omega_{\mathrm{i}}}
$$

This is the same asymptotic result previously obtained from the four-wave mixing perturbation analysis [26], which was applied in the weak injection limit without requiring the two-wavelength approximation. For $\Omega_{\mathrm{i}}<\Omega_{\mathrm{r}}$, the optical spectrum becomes nearly double sideband [26]. The two-wavelength approximation is inapplicable so that the perturbation analysis is preferred.

On the other hand, the laser under period-one oscillation eventually becomes stably locked when the injection strength increases pass the Hopf bifurcation point. At the bifurcation point, the oscillation vanishes and $a_{\mathrm{p}}$ reduces to zero. If $b \Omega_{\mathrm{r}}^{2} \gg 2\left(\gamma_{\mathrm{s}}+\gamma_{\mathrm{n}}\right) \Omega_{\mathrm{s}}$ is assumed based on typical values, then (19) implies that $\left|a_{\mathrm{i}}\right| \approx 1$. Thus, according to (15), the oscillation frequency right at the bifurcation point is

$$
\Omega_{0}=\frac{\Omega_{\mathrm{i}}+\sqrt{\Omega_{\mathrm{i}}^{2}+2\left(1+b^{2}\right) \gamma_{\mathrm{c}} \gamma_{\mathrm{n}}}}{2}
$$

for $\Omega_{0} \gg \gamma$. Since the bifurcation point is the boundary between period-one oscillation and stable locking, our analysis is compared to the eigenvalue analysis on stable locking [24]. For instance, when $\Omega_{\mathrm{i}}=0$ and $\gamma_{\mathrm{p}}=0$, our result reduces to $\Omega_{0}=\sqrt{\left(1+b^{2}\right) / 2} \Omega_{\mathrm{r}}$, which is exactly the same result according to [24]. Therefore, our analysis agrees with the established results of both four-wave mixing and stable locking.

Our analytical results are also verified against numerical results when the laser dynamical parameters are varied. Fig. 9 shows the analytical and numerical results in solid curves and data points, respectively. The frequency $\Omega_{0}$ is varied as different parameters are varied from the original values in Table I. 
Only one parameter is varied at a time. The injection is kept at $\left(\xi_{\mathrm{i}}, \Omega_{\mathrm{i}}\right)=(0.32,2 \pi \times 30 \mathrm{GHz})$. The change is applied on $b$, $\gamma_{\mathrm{c}}, \gamma_{\mathrm{n}}, \gamma_{\mathrm{s}}, \gamma_{\mathrm{p}}$, and $\tilde{J}$ for the open circles, open triangles, open squares, closed circles, closed triangles, and closed squares, respectively. It is observed that $\Omega_{0}$ is most sensitive to $\gamma_{c}$ while it is nearly insensitive to $\gamma_{\mathrm{s}}$ and $\gamma_{\mathrm{p}}$. The frequency is also independent on $\tilde{J}$ in itself. However, in reality, changing the bias always causes changes in $\gamma_{n}$ and thus changes in $\Omega_{0}$.

\section{CONCLUSION}

The nonlinear dynamical high-speed period-one oscillation of an optically injected semiconductor laser is analyzed theoretically. The formalism is based on the approximation that the laser outputs mainly two wavelengths, which correspond to the regeneration of the injection and its oscillation sideband near the shifted cavity resonance wavelength. Analytical results are expressed in terms of the injection detuning frequency and the oscillation frequency. Optical spectrum, microwave spectrum, and injection requirements of the period-one oscillation are characterized. Most interestingly, the analysis clarifies the role of the cavity resonance frequency shift, which has long been speculated without much analytical explanation. Hence, the period-one oscillation can be viewed as a generalization of four-wave mixing with the cavity resonance being shifted by strong injection. The analysis agrees well with numerical simulation on the period-one oscillation. It also bridges the theories on stable locking and four-wave mixing. The results pave the way for using the period-one oscillation as a microwave photonic source.

\section{REFERENCES}

[1] R. Lang, "Injection locking properties of a semiconductor laser," IEEE J. Quantum Electron., vol. 18, pp. 976-983, 1982.

[2] F. T. Arecchi, G. L. Lippi, G. P. Puccioni, and J. R. Tredicce, "Deterministic chaos in laser with injected signal," Opt. Commun., vol. 51, pp. 308-314, Oct. 1984.

[3] J. R. Tredicce, F. T. Arecchi, G. L. Lippi, and G. P. Puccioni, "Instabilities in lasers with an injected signal," J. Opt. Soc. Am. B, vol. 2, pp. 173-183, Jan. 1985.

[4] I. Petitbon, P. Gallion, G. Debarge, and C. Chabran, "Locking bandwidth and relaxation oscillations of an injection-locked semiconductor laser," IEEE J. Quantum Electron., vol. QE-24, pp. 148-154, Feb. 1988.

[5] G. C. Dente, P. S. Durkin, K. A. Wilson, and C. E. Moeller, "Chaos in the coherence collapse of semiconductor lasers," IEEE J. Quantum Electron., vol. QE-24, pp. 2441-2447, 1988.

[6] T. B. Simpson, J. M. Liu, K. F. Huang, and K. Tai, "Nonlinear dynamics induced by external optical injection in semiconductor lasers," Quantum Semiclass. Opt., vol. 9, pp. 765-784, Oct. 1997.

[7] S. Wieczorek, B. Krauskopf, and D. Lenstra, "A unifying view of bifurcations in a semiconductor laser subject to optical injection," Opt. Commun., vol. 172, pp. 279-295, Dec. 1999.

[8] T. B. Simpson and F. Doft, "Double-locked laser diode for microwave photonics applications," IEEE Photon. Technol. Lett., vol. 11, pp. 1476-1478, Nov. 1999.

[9] S. Wieczorek, T. B. Simpson, B. Krauskopf, and D. Lenstra, "Global quantitative predictions of complex laser dynamics," Phys. Rev. E, vol. 65, p. 045207R, Apr. 2002.

[10] T. B. Simpson, J. M. Liu, and A. Gavrielides, "Bandwidth enhancement and broadband noise reduction in injection-locked semiconductor lasers," IEEE Photon. Technol. Lett., vol. 7, pp. 709-711, 1995.

[11] E. K. Lau, H. K. Sung, and M. C. Wu, "Frequency response enhancement of optical injection-locked lasers," IEEE J. Quantum Electron., vol. 44, no. 1, pp. 90-99, Jan. 2008.

[12] F. Y. Lin and J. M. Liu, "Chaotic lidar," IEEE J. Sel. Topics Quantum Electron., vol. 10, pp. 991-997, Sep./Oct. 2004.
[13] S. C. Chan and J. M. Liu, "Tunable narrow-linewidth photonic microwave generation using semiconductor laser dynamics," IEEE J. Sel. Topics Quantum Electron., vol. 10, pp. 1025-1032, Sep./Oct. 2004.

[14] S. C. Chan, S. K. Hwang, and J. M. Liu, "Period-one oscillation for photonic microwave transmission using an optically injected semiconductor laser," Opt. Express, vol. 15, pp. 14,921-14,935, Oct. 2007.

[15] S. K. Hwang, H. F. Chen, and C. Y. Lin, "All-optical frequency conversion using nonlinear dynamics of semiconductor lasers," Opt. Lett., vol. 34, pp. 812-814, 2009.

[16] S. C. Chan, S. K. Hwang, and J. M. Liu, "Radio-over-fiber AM-to-FM upconversion using an optically injected semiconductor laser," Opt. Lett., vol. 31, pp. 2254-2256, Aug. 2006.

[17] R. Diaz, S. C. Chan, and J. M. Liu, "Lidar detection using a dual-frequency source," Opt. Lett., vol. 31, pp. 3600-3602, Dec. 2006.

[18] A. Gavrielides, V. Kovanis, T. Erneux, and M. Nizette, "Phase locked modulations of optically injected laser diodes," in SPIE, 2000, vol. 3944, pp. 627-638.

[19] T. Erneux, V. Kovanis, A. Gavrielides, and P. M. Alsing, "Mechanism for period-doubling bifurcation in a semiconductor laser subject to optical injection," Phys. Rev. A., vol. 53, pp. 4372-4380, Jun. 1996.

[20] M. Nizette, T. Erneux, A. Gavrielides, V. Kovanis, and T. B. Simpson, "Bistability of pulsating intensities for double-locked laser diodes," Phys. Rev. E, vol. 65, p. 056610, May 2002.

[21] S. Banerjee, P. Saha, and A. R. Chowdhury, "Optically injected laser system: Characterization of chaos, bifurcation, and control," Chaos, vol. 14, pp. 347-357, 2004.

[22] P. C. DeJagher, W. A. van der Graaf, and D. Lenstra, "Relaxation-oscillation phenomena in an injection-locked semiconductor laser," Quantum Semiclass. Opt., vol. 8, pp. 805-822, Aug. 1996.

[23] B. Krauskopf, N. Tollenaar, and D. Lenstra, "Tori and their bifurcations in an optically injected semiconductor laser," Opt. Commun., vol. 156, pp. 158-169, Nov. 1998.

[24] T. B. Simpson, J. M. Liu, and A. Gavrielides, "Small-signal analysis of modulation characteristics in a semiconductor laser subject to strong optical injection," IEEE J. Quantum Electron., vol. 32, no. 8, pp. 1456-1468, Aug. 1996.

[25] A. Murakami, K. Kawashima, and K. Atsuki, "Cavity resonance shift and bandwidth enhancement in semiconductor lasers with strong light injection," IEEE J. Quantum Electron., vol. 39, no. 10, pp. 1196-1204, Oct. 2003.

[26] J. M. Liu and T. B. Simpson, "Four-wave mixing and optical modulation in a semiconductor laser," IEEE J. Quantum Electron., vol. 30, no. 4, pp. 957-965, Apr. 1994.

[27] S. K. Hwang, J. M. Liu, and J. K. White, "35-GHz intrinsic bandwidth for direct modulation in 1.3- $\mu \mathrm{m}$ semiconductor lasers subject to strong injection locking," IEEE Photon. Technol. Lett., vol. 16, pp. 972-974, Apr. 2004.

[28] J. M. Liu, Photonic Devices. Cambridge, U.K.: Cambridge Univ. Press, 2005.

[29] T. B. Simpson and J. M. Liu, "Phase and amplitude characteristics of nearly degenerate four-wave mixing in Fabry-Perot semiconductor lasers," J. Appl. Phys., vol. 73, pp. 2587-2589, Mar. 1993.

[30] S. K. Hwang and J. M. Liu, "Dynamical characteristics of an optically injected semiconductor laser," Opt. Commun., vol. 183, pp. 195-205, Sep. 2000.

[31] N. M. Al-Hosiny, I. D. Henning, and M. J. Adams, "Correlation of electron density changes with optical frequency shifts in optically injected semiconductor lasers," IEEE J. Quantum Electron., vol. 42, pp. 570-580, 2006.

[32] T. B. Simpson, "Phase-locked microwave-frequency modulations in optically-injected laser diodes," Opt. Commun., vol. 170, pp. 93-98, Oct. 1999.

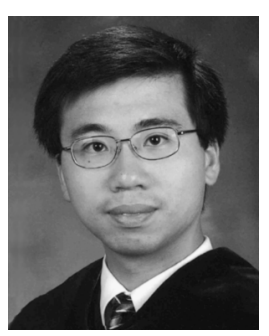

Sze-Chun Chan received the B.Eng. degree in electrical and electronic engineering from the University of Hong Kong, China, in 2001, and the M.S. and $\mathrm{Ph} . \mathrm{D}$. degrees in electrical engineering from the University of California at Los Angeles (UCLA) in 2004 and 2007, respectively. He received the Dr. Bor-Uei Chen Scholarship of the Photonics Society of Chinese-Americans in 2007.

$\mathrm{He}$ is currently an Assistant Professor of electronic engineering at the City University of Hong Kong. His research interests include laser nonlinear dynamics, microwave photonics, radio-over-fiber, optical chaos generation, and biological applications of semiconductor lasers. 